\title{
Erratum to: Stable periodicity and negative circuits in differential systems
}

\author{
Adrien Richard · Jean-Paul Comet
}

Published online: 14 January 2015

(C) Springer-Verlag Berlin Heidelberg 2015

\section{Erratum to: J. Math. Biol. (2011) 63:593-600 DOI 10.1007/s00285-010-0388-y}

In a private communication, Frederic Beck (University of Mainz) pointed out that, in the originally published article, when proving that $\Omega$ contains a stable periodic solution we use a wrong argument, the following: $\left(\partial f_{i} / \partial x_{i}\right)(x)<0$ for all $x \in \Omega, i=1,2$. Indeed, for instance, if $\left.x_{1} \in\right] 2-\varepsilon, 2+\varepsilon\left[\right.$ and $\left.x_{2} \in \times[1+\varepsilon, 3-\varepsilon] \backslash\right] 2-\varepsilon, 2+\varepsilon[$ then $\left(\partial f_{i} / \partial x_{i}\right)(x)=4 \varphi_{2}^{\prime}\left(x_{1}\right)-1$, and this term can not be less than zero for all $\left.x_{1} \in\right] 2-\varepsilon, 2+\varepsilon$ [ because $\varphi_{2}^{\prime}$ has to be greater than $1 / 2 \varepsilon>1$ somewhere in this region. So there are $x \in \Omega$ with $\left(\partial f_{i} / \partial x_{i}\right)(x)>0$.

However, using slightly more involved arguments, we proved here that the system is still a counter-example of Conjecture $2^{\prime}$. More precisely, we prove that if $\varepsilon \leq 1 / 8$ then there is a stable periodic solution in the domain $\Omega^{\prime}=[0,4]^{2} \backslash \Gamma$, where $\Gamma$ is the interior of the convex hull of the set containing the points $A=(1-\varepsilon, 3-\varepsilon)$, $B=(2-\varepsilon, 3+\varepsilon), C=(3-\varepsilon, 3+\varepsilon), D=(3+\varepsilon, 2+\varepsilon), E=(3+\varepsilon, 1+\varepsilon)$, $F=(2+\varepsilon, 1-\varepsilon), G=(1+\varepsilon, 1-\varepsilon)$, and $H=(1-\varepsilon, 2-\varepsilon)$; see Fig. 1 for an illustration.

First, since $\Omega^{\prime} \subseteq \Omega$, there is no equilibrium point in $\Omega^{\prime}$. Suppose now that $\varepsilon \leq 1 / 8$, and let us prove that all the solutions starting in $\Omega^{\prime}$ remain in $\Omega^{\prime}$. As showed in the originally published article, no solution starting in $[0,4]^{2}$ leaves $[0,4]^{2}$, thus it is

The online version of the original article can be found under doi:10.1007/s00285-010-0388-y.

A. Richard $(\varangle) \cdot$ J.-P. Comet

13S, UMR 6070 CNRS, Université de Nice-Sophia Antipolis, 2000 route des Lucioles, 06903 Sophia Antipolis, France

e-mail: richard@unice.fr

J.-P. Comet

e-mail: comet@unice.fr 
Fig. 1 The gray region is an illustration of $\Omega^{\prime}$

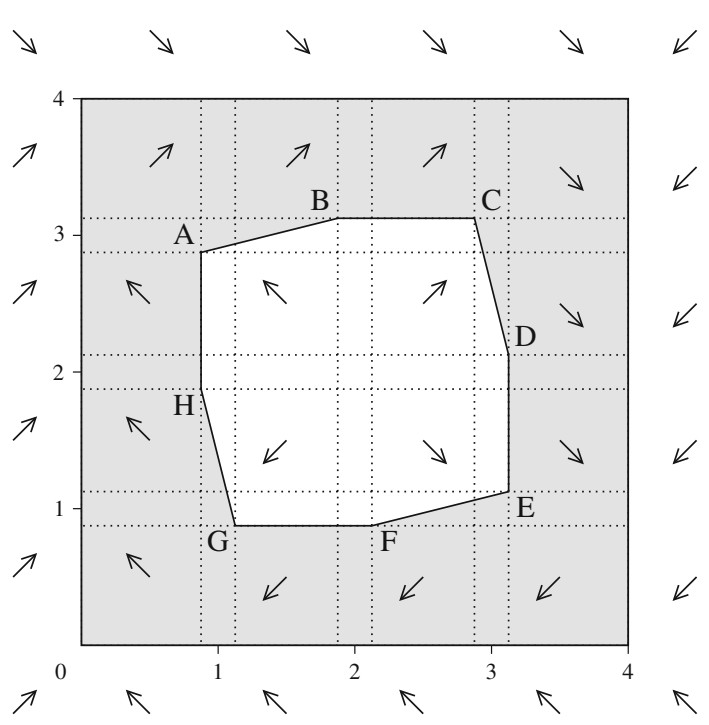

sufficient to prove that no solution starting in $\Omega^{\prime}$ reaches the interior of the convex hull $\Gamma$. Consider first the line segment $L$ with endpoints $A$ and $B$. For all $x \in L$ we have

$$
\begin{aligned}
& f_{1}(x)=4 \varphi_{3}\left(x_{2}\right)-x_{1} \leq 4-x_{1} \leq 3+\varepsilon \\
& f_{2}(x)=4-x_{2} \geq 1-\varepsilon .
\end{aligned}
$$

Thus for all $x \in L$ the scalar product between $f(x)$ and the vector $v=(-2 \varepsilon, 1)$ is at least $-2 \varepsilon(3+\varepsilon)+(1-\varepsilon)=1-7 \varepsilon-2 \varepsilon^{2}$, and this term is positive since $\varepsilon \leq 1 / 8$. Since $v$ is orthogonal to $L$ and is pointing outside $\Gamma$, this means that if a solution starts in $\Omega^{\prime}$, then it cannot reach $\Gamma$ by crossing the line segment $L=A B$. Also, for all $x$ that lies in the line segment $B C$ we have $f_{2}(x)=1-\varepsilon>0$. Thus if a solution starts in $\Omega^{\prime}$, then it cannot reach $\Gamma$ by crossing $B C$. Reasoning similarly with the segments $C D, D E, E F, F G, G H$, and $H A$, we deduce that all the solutions starting in $\Omega^{\prime}$ remains in $\Omega^{\prime}$. Thus, following the Poincaré-Bendixon theorem, there exists a periodic solution $\psi$ of period $T>0$ starting in $\Omega^{\prime}$.

Finally, let us prove that $\psi$ is stable. For all $x \in \mathbb{R}^{2}$ we have $\left(\partial f_{1}(x) / \partial x_{1}\right)(x)=$ $4 \varphi_{2}^{\prime}\left(x_{1}\right)\left(\varphi_{1}\left(x_{2}\right)-\varphi_{3}\left(x_{2}\right)\right)-1$. Thus $\left(\partial f_{1}(x) / \partial x_{1}\right)(x) \geq 0$ implies $4 \varphi_{2}^{\prime}\left(x_{1}\right)\left(\varphi_{1}\left(x_{2}\right)-\right.$ $\left.\varphi_{3}\left(x_{2}\right)\right)>0$ which implies that $x$ belongs to the domain $] 2-\varepsilon, 2+\varepsilon[\times] 1-\varepsilon, 3+\varepsilon[$ which is disjoint from $\Omega^{\prime}$. Thus $\left(\partial f_{1} / \partial x_{1}\right)(x)<0$ for all $x \in \Omega^{\prime}$, and we prove with similar arguments that $\left(\partial f_{2} / \partial x_{2}\right)(x)<0$ for all $x \in \Omega^{\prime}$. Thus

$$
\int_{0}^{T} \frac{\partial f_{1}}{\partial x_{1}}(\psi(t))+\frac{\partial f_{2}}{\partial x_{2}}(\psi(t)) \mathrm{d} t<0
$$

and we deduce that $\psi$ is stable. 